\title{
Expression patterns of HENMT1 and PIWIL1 in human testis: implications for transposon expression
}

\author{
A L Hempfling 1,2, S L Lim , D L Adelson ${ }^{3}$, J Evans ${ }^{4}$, A E O'Connor ${ }^{1}$, Z P Qu ${ }^{3}$, S Kliesch ${ }^{5}$, \\ W Weidner ${ }^{6}, \mathrm{M} \mathrm{K} \mathrm{O}^{\prime}$ Bryan $^{1,7}$ and M Bergmann ${ }^{2}$ \\ ${ }^{1}$ The Development and Stem Cells Program of the Monash Biomedicine Discovery Institute and The Department of \\ Anatomy and Developmental Biology, Monash University Clayton, Clayton, Australia, ${ }^{2}$ Institute for Veterinary \\ Anatomy, Histology and Embryology, Justus Liebig University, Giessen, Germany, ${ }^{3}$ School of Biological Sciences, \\ The University of Adelaide, Adelaide, Australia, ${ }^{4}$ Centre for Reproductive Health, Hudson Institute of Medical \\ Research, Clayton, Australia, ${ }^{5}$ Centre of Reproductive Medicine and Andrology, Muenster, Germany, ${ }^{6}$ Clinic for \\ Urology, Pediatric Urology and Andrology, Justus-Liebig-University, Giessen, Germany and ${ }^{7}$ The School of Biological \\ Sciences, Monash University, Clayton, Australia \\ Correspondence should be addressed to A-L Hempfling; Email: Anna-Lena.Hempfling@vetmed.uni-giessen.de
}

\begin{abstract}
This study aimed to define the expression patterns of HENMT1 and PIWI proteins in human testis and investigate their association with transposon expression, infertility sub-type or development of testicular germ cell tumours (TGCTs). Testis biopsies showing normal spermatogenesis were used to identify normal localisation patterns of HENMT1 and PIWIL1 by immunolocalisation and RT-PCR after laser microdissection. 222 testis biopsies representing normal spermatogenesis, hypospermatogenesis, spermatogenic arrests, Sertoli cell-only (SCO) tumours and TGCTs were analysed by RT-qPCR for expression of HENMT1/PIWIL1/PIWIL2/PIWIL3/ PIWIL4 and LINE-1. Additionally, HENMT1-overexpressing TCam2 seminoma cell lines were analysed for the same parameters by RT-qPCR. We found that HENMT1 and PIWIL1 are coexpressed in pachytene spermatocytes and spermatids. Expression of HENMT1, PIWIL1 and PIWIL2 was mainly dependent on germ cell content but low levels of expression were also detected in some SCO samples. Levels of HENMT1, PIWIL1 and PIWIL2 expression were low in TGCT. Samples with HENMT1, PIWIL2 and PIWIL4 expression showed significantly $(P<0.05)$ lower transposon expression compared to samples without expression in the same histological group. HENMT1-overexpressing TCam2 cells showed lower LINE-1 expression than empty vector-transfected control lines. Our findings support that the transposon-regulating function of the piRNA pathway found in the mouse is conserved in adult human testis. HENMT1 and PIWI proteins are expressed in a germ-cell-specific manner and required for transposon control.

Reproduction (2017) 154 363-374
\end{abstract}

\section{Introduction}

Human male infertility and testis cancer show a growing incidence and are increasingly considered to be interconnected as part of a 'testicular dysgenesis syndrome' (Skakkebaek 2016). Despite this, the mechanisms underlying both pathologies are not well understood, thus hampering prevention and treatment. Testis cancer is the most common form of cancer in young men; while it responds well to treatment if discovered early, significant side effects and treatmentresistant cases pose a problem (Nitzsche et al. 2012). On the other hand, many infertility patients will never find out why their spermatogenesis is impaired and will never be able to father their own biological offspring. One essential prerequisite for successful spermatogenesis is the control of mobile genomic elements called retrotransposons in germ cells by methylation or post-transcriptional repression (Kuramochi-Miyagawa et al. 2008, Reuter et al. 2011a, Zamudio et al. 2015). Retrotransposons are mobile DNA elements which are able to move around in the genome by a 'copy and paste' mechanism using enzymes encoded in their own, or other retrotransposons', sequence. Failure to suppress retrotransposon replication frequently results in damage to the genome, such as double strand breaks and mutations, leading to infertility or birth defects in offspring. This is especially important during the phase of genome-wide hypomethylation and the zygotene-pachytene transition where other transposon control mechanisms, like DNA methylation and repressive histone marks, are reduced or temporarily inactive (Bourc'his \& Bestort 2004, Webster et al. 2005, Di Giacomo et al. 2013).

The PIWI/piRNA pathway is an ancient pathway first identified in Arabidopsis (Chen et al. 2002, Yu et al. 2005) 
and is recognised as an important mechanism that protects the germ cell genome from retrotransposon insertional mutagenesis.

piRNAs are short noncoding RNAs that bind to PIWI proteins (Girard et al. 2006), a subfamily of the PIWI/ Argonaute protein family with a distinct RNA cleaving activity (Lau 2006). Primary piRNAs are encoded as long precursor transcripts transcribed from piRNA genes (Williams et al. 2015). They are exported into the cytoplasm, where the endonuclease MITOPLD/ PLD6 generates the $5^{\prime}$ ends of what will ultimately be piRNAs (Watanabe et al. 2011). These are subsequently loaded onto PIWIL1 or PIWIL2 (Kawaoka et al. 2011, Vourekas et al. 2012). The 3' end of the piRNA is generated by an unknown endonuclease, and subsequently methylated by HENMT1 (HUA Enhancer 1) (Kirino \& Mourelatos 2007, Saito et al. 2007, Plotnikova et al. 2013), producing a mature primary piRNA. This piRNA can then direct PIWI proteins to target complementary mRNA sequences which are subsequently cleaved.

Secondary piRNAs are produced via the ping-pong cycle (Brennecke et al. 2007, Aravin et al. 2008), a feed-forward mechanism involving PIWIL2 and PIWIL4, which is recognised as an essential 'adaptive immune system' to destroy transposon transcripts in foetal germ cells and spermatogonia and has been proposed to exist in adult germ cells as well (Ha et al. 2014, Goh et al. 2015, Lim et al. 2015, Rounge et al. 2015). In addition to this post-transcriptional mechanism, piRNAs are thought to build an epigenetic defence layer against transposon expression by directing the methylation of $\mathrm{CpG}$ islands and histones (Carmell et al. 2007, Di Giacomo et al. 2013, Guo \& Wu 2013).

Mammals express two distinct populations of piRNAs, a more transposon-enriched group in pre-pachytene germ cells and a transposon-depleted pachytene piRNA population, more enriched for intergenic regions (Aravin et al. 2007, Brennecke et al. 2007, Lim et al. 2015). At least a portion of the latter is thought to be involved in controlling the spermatogenic expression programme, as well as transposon expression, and the removal of excess mRNA in the last steps of spermatogenesis (Gou et al. 2014, Lim et al. 2015).

A recent paper investigating a Henmt 1 mouse mutant demonstrated that the methyltransferase is essential for mouse spermatogenesis (Lim et al. 2015). Lack of HENMT1 led to piRNA instability due to reduced methylation of the piRNA 3' end, the de-repression of retrotransposons in meiotic and haploid germ cells, and the precocious expression of the haploid germ cell programme in meiotic cells, resulting in malformed spermatids and male infertility.

HENMT1 was shown to be produced in all germ cells of the seminiferous epithelium in the mouse with highest expression in late pachytene spermatocytes and spermatids (Lim et al. 2015).
The mouse PIWIL1 homolog, MIWI, is detected specifically in the cytoplasm of spermatocytes and spermatids and is essential for spermatogenesis (Deng \& Lin 2002). Miwi mutant mice show an arrest of spermatogenesis during the round spermatid period. In addition to MIWI, mice also express the PIWI proteins MILI (PIWIL2) and MIWI2 (PIWIL4). MILI is produced in the cytoplasm of germ cells from the primordial germ cell stage to the round spermatids stage and mutants are male sterile, with normal primordial germ cell development but an arrest at the zygotene-pachytene transition (Kuramochi-Miyagawa et al. 2004, Aravin et al. 2008). MIWI2 is the only mouse PIWI protein localizing to both nucleus and cytoplasm. It is only detected in primordial germ cells and prospermatogonia between E12.5 and P3 (Aravin et al. 2008, Bao et al. 2014), corresponding to the period of de novo DNA methylation. Miwi2 knockout in mice resulted in demethylation of LINE-1 (L1) elements, a pre-pachytene arrest of spermatogenesis, as well as a progressive loss of germ cells that ultimately resulted in tubules containing only Sertoli cells (Carmell et al. 2007).

In summary, the localisation and function of HENMT1 and PIWI proteins in mouse spermatogenesis has been investigated extensively while the spatio-temporal expression of their human homologues is still largely unknown. Additionally, an association of Piwi proteins has also been discovered in different cancer types (Suzuki et al. 2012, Wang et al. 2015, Iliev et al. 2016).

To improve understanding of the PIWI/piRNA pathway in human testis, this study aimed to define the expression patterns of HENMT1 and PIWIL1 in the human testis and investigate whether changes in their expression were associated with transposon de-repression, particular types of human infertility or TGCTs.

\section{Materials and methods}

\section{Testis biopsies}

This study was approved by the ethics committee of the Medical Faculty of the Justus Liebig University Giessen (Decision 187b/09) and the Monash University human ethics committee. Testicular biopsies were taken, after obtaining written informed consent, at the Department for Clinical Andrology, Centre for Reproductive Medicine and Andrology in the University of Muenster or at the Department of Urology in the University Hospital Giessen (UKGM).

Testis samples were obtained from men, aged 15-74 years, who underwent diagnostic testicular biopsies as described previously (Bergmann \& Kliesch 2010). Testicular biopsies were fixed by immersion in Bouin's solution and embedded in paraffin. For histological evaluation, slides were prepared and evaluated following score countanalysis as described previously (Bergmann \& Kliesch 2010). Testicular biopsies showed normal spermatogenesis (Nsp, $n=21$ ), hypospermatogenesis (hyp, score 1-10, $n=49$ ), hypospermatogenesis with focal Sertoli cell-only (SCO) (hyp +fsc, $n=11$ ), tumours (TIN/Seminoma/ 
Carcinoma, $n=22$ ), arrest at the level of primary spermatocytes or spermatogonia (Arrests, $n=55$ ) as well as SCO syndrome $(n=65)$ (Bergmann \& Kliesch 2010).

\section{Laser assisted microdissection PCR}

Microdissection was performed as described previously (Fietz et al. 2011). Samples representing the whole seminiferous epithelium, and the seminiferous epithelium without spermatogonia, were microdissected from normal spermatogenesis. Samples representing spermatocytes or spermatogonia were dissected using tissue showing arrests at the level of spermatocytes or spermatogonia, respectively. A pure Sertoli cell population was obtained from SCO testes.

RNA extraction was accomplished with the RNeasy FFPE kit (Qiagen) according to the 'Purification of Total RNA from Microdissected FFPE Tissue Sections' protocol. After DNAse treatment, RNA was reverse transcribed with Roche Superscript III (Roche) according to manufacturer's instructions. A control PCR with GAPDH primers was performed to control for gDNA contamination using AmpliTaq Gold (Applied Biosystems) according to the manufacturer's instructions. Primer pairs used on cDNA from microdissected material were HENMT1V2 for CCAGAATGGAGTTTCAGACC and HENMT1V2 rev GATTCTGTTGCCTTTCCTCC and PIWIL1 for ACTGGTGAACTACGAAGTGC and PIWIL1 rev GGATTCTGAAGTCTTCCTCC.

\section{Immunochemistry}

The immunofluorescent labelling of proteins was conducted as described previously (Jamsai et al. 2011). The well-established primary antibodies (HENMT1 (specificity proven on knockout in (Lim et al. 2015)) and PIWIL1 (Abcam ab12337); both $5 \mu \mathrm{g} / \mathrm{mL}$ ) were incubated at $4{ }^{\circ} \mathrm{C}$ overnight. Secondary antibodies anti-goat AF 455 and anti-rabbit 455 (Life Technologies; $4 \mu \mathrm{g} / \mathrm{mL}$ ) were incubated $1 \mathrm{~h}$ at room temperature in the darkness. Sections were counterstained with DAPI and coverslipped with fluorescence mounting media (DAKO). Pictures were taken on a Leica SP8 confocal microscope (Leica).

For immunochemistry sections were labelled as described previously (Fietz et al. 2014) using the PIWIL1 antibody
(Abcam ab12337; $7 \mu \mathrm{g} / \mathrm{mL}$ ). For a negative control, the primary antibody was pre-incubated with an excess of the immunizing peptide ab13827 (Abcam). Pictures were taken with a Leica ICC50 HD Microscope Camera on a Leica DM750 light microscope (Leica).

\section{qPCR}

RNA extraction from whole Bouins-fixed tissue sections was accomplished with the RNeasy FFPE kit (Qiagen) following the 'Purification of Total RNA from FFPE Tissue Sections' protocol. cDNA was produced and a control PCR was performed as described above. $1 \mu \mathrm{L}$ cDNA was then used with TaqMan Fast Advanced Master Mix (Thermo Fisher Scientific) in realtime PCRs with TaqMan gene expression assays (Table 1) on a Biorad CFX384 Touch cycler (Biorad). Input was normalised to the housekeeping gene 18S (Table 1).

For detection of actively transposing L1 sequences, we designed TaqMan assays interrogating the active human consensus L1 sequence L1HS (Table 1). These assays were tested for specificity in an ePCR (https://www.ncbi.nlm.nih.gov/ projects/e-pcr/reverse.cgi; parameters: max. three mismatches, no gap allowed) that returned one target amplification site, which is the expected sequence across the whole human genome.

Samples were analysed in triplicate and excluded from analysis if the standard deviation between the replicates exceeded $0.6 \mathrm{Cq}$. $\mathrm{Cq}$ values $>38$ were considered as no expression/no detection. The $2^{\mathrm{DCq}}$ value was used for further analysis in GraphPad Prism.

\section{Cell culture conditions and transfection}

TCam2 cells were cultured in RPMI 1640 medium with $10 \%$ FBS and $1 \%$ penicillin-streptomycin at $37^{\circ} \mathrm{C}$. Cells were transfected with the PiggyBac Transposon Vector system (SBI System Biosciences, Palo Alto, USA) using the NEON transfection system (Thermo Fisher Scientific) according to the manufacturer's instructions with a single pulse of $1000 \mathrm{~V}$ for $40 \mathrm{~ms}$. Three cell lines were transfected with a PiggyBac vector $(6.5 \mu$ g PiggyBac vector $+2.25 \mu$ g transposase vector $/ 12 \times 10^{5}$ cells) containing the human HENMT1 full

Table 1 TaqMan gene expression assays.

\begin{tabular}{|c|c|c|}
\hline Assay name & Assay ID & RefSeq/GenBank/Repbase ID \\
\hline \multirow[t]{2}{*}{ HENMT1 } & Hs00989130_m1 & NM_144584.2592.1 \\
\hline & & NM_001102592.1 \\
\hline \multirow{2}{*}{ PIWIL1 } & Hs01041737_m1 & NM_001190971.1 \\
\hline & & NM_004764.4 \\
\hline \multirow[t]{2}{*}{ PIWIL2 } & Hs00216263_m1 & NM_001135721.1 \\
\hline & & NM_018068.3 \\
\hline \multirow[t]{4}{*}{ PIWIL3 } & Hs00908825_m1 & NM_001008496.3 \\
\hline & & NM_001255975.1 \\
\hline & & NR_045648.1 \\
\hline & & NR_045649.1 \\
\hline PIWIL4 & Hs00381509_m1 & NM_152431.2 \\
\hline $18 S$ & Hs99999901_s1 & X03205.1 \\
\hline HL1A Custom TaqMan & SM AIGJQ74 P140602-000G08 & L1HS Consensus 5'UTR \\
\hline HL1B Custom TaqMan & SM AIHSPEC P140602-000G09 & L1HS Consensus ORF1 \\
\hline
\end{tabular}


length sequence (NM_144584.2, verified by sequencing) and three cell lines with an empty PiggyBac vector (6.5 $\mu$ g PiggyBac vector $+2.25 \mu \mathrm{g}$ transposase vector $/ 12 \times 10^{5}$ cells) as a control. Additionally, one reaction was transfected with a pEGFP vector (4.4 $\mu \mathrm{g}$ pEGFP vector $/ 6 \times 10^{5}$ cells) as a transfection efficiency control. The PiggyBac vector carried a puromycin resistance gene, so transfected TCam2 cells were cultured in the medium described above plus $2 \mu \mathrm{g} / \mathrm{mL}$ puromycin to select successfully transfected cells.

RNA was purified using Trizol, and DNAse-treated and reverse-transcribed with Superscript III (Thermo Fisher Scientific). HENMT1 overexpression was confirmed by realtime PCR as described in the section ' $q P C R$ '.

\section{Statistics}

For the cell line qPCR data, the mean of three repetitions and three technical replicates per cell line (exception Hen1 \#3: only 1 repetition) and then the mean of three cell lines for empty vector and HENMT1-transfected cells respectively were calculated and the final mean compared with an unpaired, two-tailed $t$-test.

\section{Results \\ Localisation of HENMT1 and PIWIL1}

The localisation and cell-specific production of HENMT1 within the human seminiferous epithelium has not been defined before. While PIWIL1 expression has been studied in more depth, a detailed description of PIWIL1 stage specific localisation in the human testis has not been published. We showed expression of HENMT1 and PIWIL1 in human testis using
RT-PCR following laser microdissection of human testis sections and pinpointed their cell-specific expression and subcellular localisation using immunolocalisation.

Using RT-PCR on laser microdissected cell populations (Fig. 1), HENMT1 expression was found in all germ cell types (Fig. 1A, B, C and D) and isolated Sertoli cells (Fig. 1E). PIWIL1 expression was found in fractions containing spermatocytes (Fig. 1A, B and $\mathrm{C}$ ), and spermatids (Fig. $1 \mathrm{~A}$ and $\mathrm{B}$ ), and in isolated spermatocytes (Fig. 1C), but not in spermatogonia (Fig. 1D) or Sertoli cells (Fig. 1E). HENMT1 protein was shown to be localised in the cytoplasm of all stages of germ cells (spermatogonia, spermatocytes and spermatids) as well as Sertoli cells, with highest levels in spermatocytes (Fig. 2A and C).

PIWIL1 protein production was first seen in the cytoplasm of late pachytene cells in stage IV, where it was strongest, and continued on a lower level in the cytoplasm of spermatids until it disappeared in elongated spermatids of stage II (Fig. 2B, E and K). This resulted in co-localisation of HENMT1 and PIWIL1 in late pachytene until elongated spermatids (Fig. 2C and E). PIWIL1 was not detected in spermatogonia or Sertoli cells at RNA or protein level (Figs 1D, E and 2F, $\mathrm{K})$. In pachytene cells, the strongest staining for PIWIL1 was observed in irregularly formed structures in the cytoplasm (Fig. 2I inset), which likely represents the mitochondrial cement (Fawcett et al. 1970, Takebe et al. 2013). In spermatids, the strongest staining was limited to one round structure in the cytoplasm, fitting the description of the chromatoid body as a changing but clear structure in spermatids (Fawcett et al. 1970, Takebe et al. 2013).

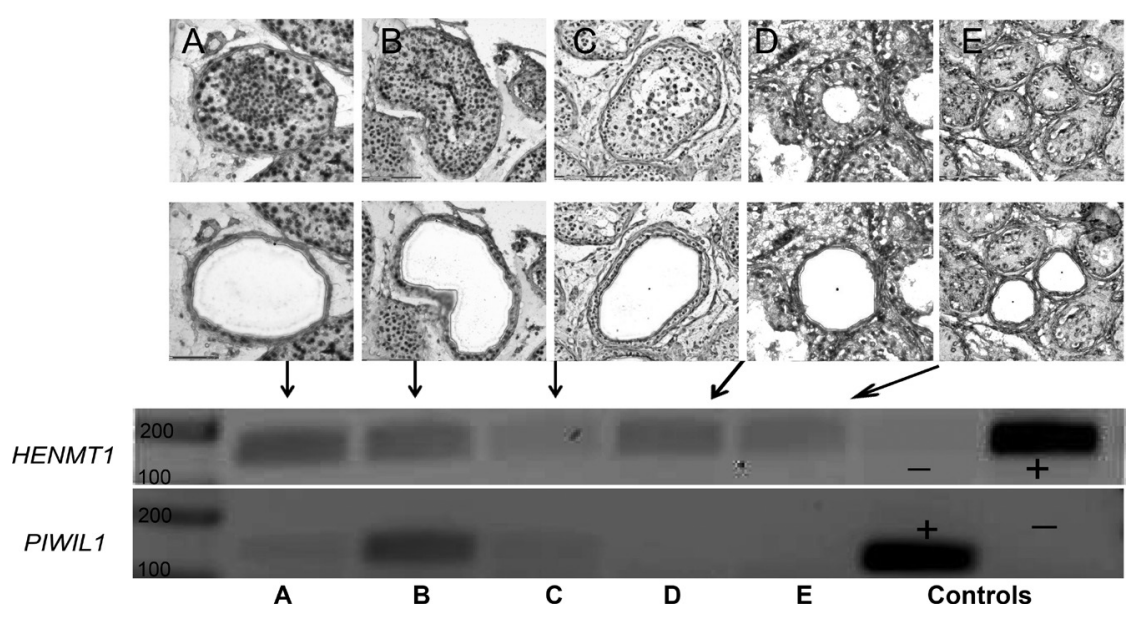

Reproduction (2017) 154 363-374
Figure 1 HENMT1 mRNA is expressed in all types of germ cells and Sertoli cells. PIWIL1 mRNA is expressed in spermatocytes and spermatids, but not in spermatogonia or Sertoli cells. HENMT1 and PIWIL1 expression analysed by RT-PCR after laser microdissection of different cell populations from paraffin sections. (A) Whole seminiferous epithelium from normal spermatogenesis. (B) Seminiferous epithelium without spermatogonia from normal spermatogenesis. (C) Spermatocytes from testis showing arrest at the stage of spermatocytes. (D) Spermatogonia from testis showing arrest at the stage of spermatogonia. (E) Sertoli cells from SCO. Arrows point from section to the respective PCR band, and the PCR bands have been labelled with matching letters. The last two PCR bands show the positive $(+)$ and no template control (-) respectively. The first lane represents the DNA marker (bands $100 \mathrm{bp}$ and $200 \mathrm{bp}$ ). PCR product sizes are $159 \mathrm{bp}$ for HENMT1 and 143 bp for PIWIL1. 

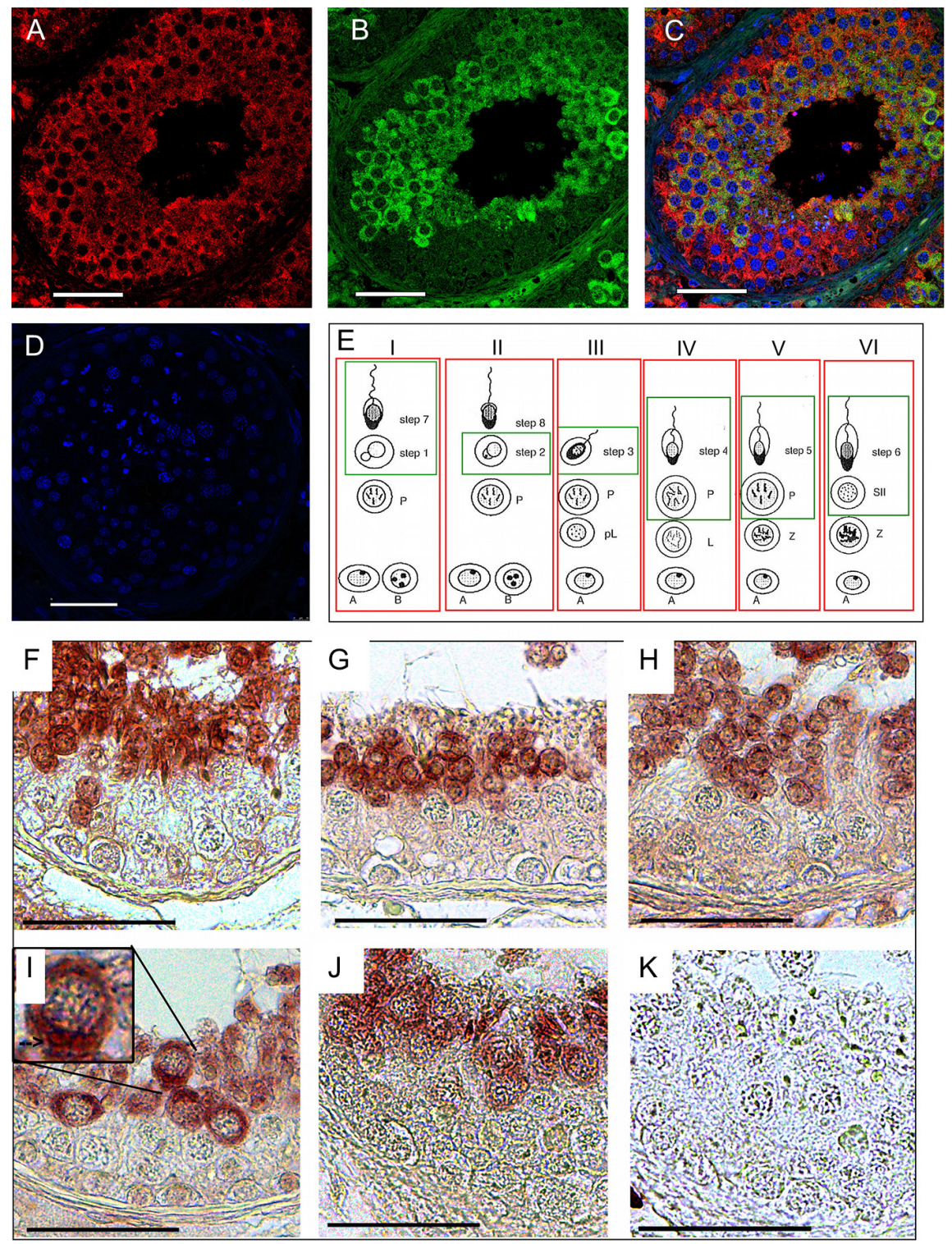

Figure 2 HENMT1 and PIWIL1 are produced in human testis and colocalise in pachytene spermatocytes and spermatids. (A) HENMT1 localisation in all cells of the seminiferous epithelium (red). (B) PIWIL1 localisation (green) in late pachytene spermatocytes and spermatids. (C) Colocalisation of HENMT1 and PIWIL1 in pachytene spermatocytes and spermatids (merge). (D) Negative control (secondary antibodies AF $555+488$ only) merge. Scale bars $=50 \mu \mathrm{m}$. (E) Stages I-VI corresponding to $\mathrm{F}-\mathrm{K}$ with cells showing PIWIL1 immunoreactivity marked by green, and cells showing HENMT1

immunoreactivity marked by red boxes. ( $F, G, H, I$ and J) Immunohistochemistry: PIWIL1 localisation in different steps (pachytene to step 7) and stages (I (F), II (G), III (H), IV (I), V (J)) of the human seminiferous epithelium, (Inset I) pachytene spermatocyte with irregular structure in cytoplasm (arrow). (K) Negative control (pre-incubation of antibody with peptide). Scale bars $=50 \mu \mathrm{m}$.

\section{piRNA pathway components are differentially expressed in histological groups}

To get a picture of the expression of the piRNA pathway and retrotransposon sequences in human testis and how they relate to each other, we quantified the expression of HENMT1, PIWIL1, PIWIL2, PIWIL3 and PIWIL4, and L1 (LINE-1) in human testis biopsies. L1 was selected as the retrotransposon family of choice as they are significantly de-repressed in numerous mouse models of piRNA pathway dysfunction (Carmell et al. 2007, Fazio et al. 2011, Lim et al. 2015) and retrotransposition events due to L1 and Alu are most common in human genomes. DNA transposons and LTR retrotransposons do not play as significant a role in generating human mobile element insertions, and Alu retrotransposons are dependent on L1 ORF2 (International Human
Genome Sequencing Consortium 2001). L1s make up $\sim 17 \%$ of the human genome (International Human Genome Sequencing Consortium 2001) and another $\sim 17 \%$ of the genome are comprised of Alu elements $(10 \%)$ and processed pseudogenes mobilised with the help of $L 1$ (Esnault et al. 2000, Symer et al. 2002). Most L1 copies are ancient/inactive and/or $5^{\prime}$ truncated and thus inactive so L1 expression is a function of younger, actively transposing ('hot') L1 A and B.

HENMT1 was detected in $81 \%$ of Nsp samples, $82 \%$ of hypospermatogenesis (Hyp) samples, $80 \%$ of arrest samples, $55 \%$ of tumour samples and $25 \%$ of SCO samples. Taken as a group, expression was highest in Nsp. Expression was halved in samples containing Hyp and became progressively lower in Hyp $+\mathrm{fsc}$ and arrest groups (Fig. 3). The lowest expression in all groups was found in SCO samples. These data suggest that 


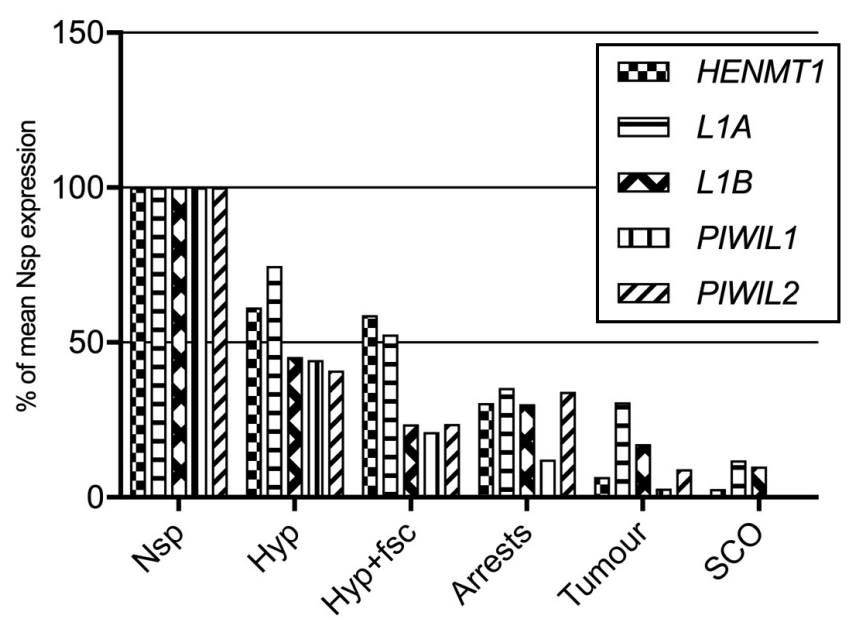

Figure 3 Expression of piRNA pathway genes and TEs decreases with reduced germ cell content in samples. Relative expression (per cent of mean Nsp expression) of HENMT1, L1A, L1B, PIWIL1 and PIWIL2 in the different histological groups. Only samples expressing HENMT1 included. Nsp $n=17$; Hyp $n=40 ;$ Hyp + fsc $n=7$; Arrests $n=40$; Tumour $n=12$; SCO $n=16$.

HENMT1 is predominantly produced by germ cells and is consistent with germ cells being the primary site of piRNA actions in mice.

PIWIL 1 was expressed in all Nsp and Hyp samples and $96 \%$ of arrest samples, but only in 5\% of SCO samples. Expression was detected in $70 \%$ of tumour tissues which was unexpected as PIWIL1 is not normally expressed in gonocytes or spermatogonia, from which germ cell tumours are thought to be derived (Jorgensen et al. 1995). The highest expression was again found in Nsp samples, with Hyp samples showing less than half of the Nsp expression and expression in Hyp $+\mathrm{fsc}$ and arrest groups being below $20 \%$ and $10 \%$ respectively (Fig. 3). Expression in tumour and SCO groups was very low.

PIWIL2 was expressed in all Nsp/Hyp, 98\% of arrest samples and $95 \%$ of tumour samples, but also in $56 \%$ of SCO samples, suggesting less strict germcell-specific expression than PIWIL1. However, PIWIL2 levels were very low in SCO samples (Fig. 3). Expression was highest in Nsp samples and reduced to a similar degree as PIWIL1 in Hyp and Hyp + fsc groups (Fig. 3). In the arrest group, however, PIWIL2 levels were higher than in Hyp samples. In arrest and tumour samples, we found a reduction compared to Nsp expression for PIWIL2, but it is less pronounced than that for PIWIL1. Within the tumour group, we could not identify significant differences in expression of transposon sequences or piRNA pathway components between TIN, carcinoma and seminoma (Supplementary Fig. 1, see section on supplementary data given at the end of this article).

PIWIL3 was not detected in any of the 94 human testis biopsies examined. PIWIL4 expression was exclusively analysed in the arrest group and detected in $91 \%$ of samples.

$L 1$ ( $A$ and $B$ ) transcripts were expressed in all patients. Surprisingly, their mean expression was highest in Nsp and reduced in Hyp, Hyp + fsc and arrest samples in a pattern similar to the piRNA pathway components. The high expression in some Nsp samples was unexpected, as retrotransposons are not thought to be significantly expressed during normal spermatogenesis. In contrast to the other groups where $L 1$ and piRNA components were reduced in a similar manner, the mean $\angle 1$ expression in the tumour samples seemed to be higher in relation to all three piRNA pathway components.

\section{HENMT1 expression is inversely correlated with $L 1$ expression in human testis}

Since HENMT1 expression was not detectable in all testis biopsies, we investigated an association between HENMT1 expression and the expression of transposons and other piRNA pathway components in human testis by comparing the L1, PIWIL1, PIWIL2 and PIWIL4 expression in samples with and without HENMT1 expression. The $L 1$ expression was significantly higher in samples without HENMT1 expression in all six histological groups, suggesting a suppressive effect of HENMT1 expression on transposon expression in human testis (Fig. 4A).

Interestingly, PIWIL1 levels were also higher in samples without HENMT1 expression in Nsp/Hyp and in the arrest group (Fig. 4A). The correlation between PIWIL1 and L1 expression was very strong in the Nsp/ Hyp group (Spearman $r=0.94$ ). It was much weaker in all other groups, probably because they contained much lower quantities of the cell types normally expressing PIWIL1.

Surprisingly, the expression associations of $L 1$ and piRNA pathway components in SCO testes were quite similar to samples containing germ cells, albeit at much lower levels (Fig. 3). L1s were expressed at significantly higher levels in SCO samples without HENMT1 expression (Fig. 4A).

As with PIWIL1 and L1, PIWIL2 expression was increased in the Nsp/Hyp group lacking HENMT1 expression (Fig. 4A). The correlation between PIWIL2 and $L 1$ expression was very strong in Nsp/Hyp $(r=0.95)$. It was slightly weaker $(r=0.9)$ in the arrest group and dropped to 0.6 in the tumour samples.

We also compared retrotransposon expression in SCO samples with $(n=37)$ or without $(n=28)$ PIWIL2 expression. Samples without PIWIL2 expression were found to have a significantly higher $L 1 A$ expression than samples which had detectable PIWIL2 expression (Fig. 4B). However, the majority of these samples also had no HENMT1 expression so we compared samples lacking both PIWIL2 and HENMT1 with samples lacking only one piRNA component. We found that 
A
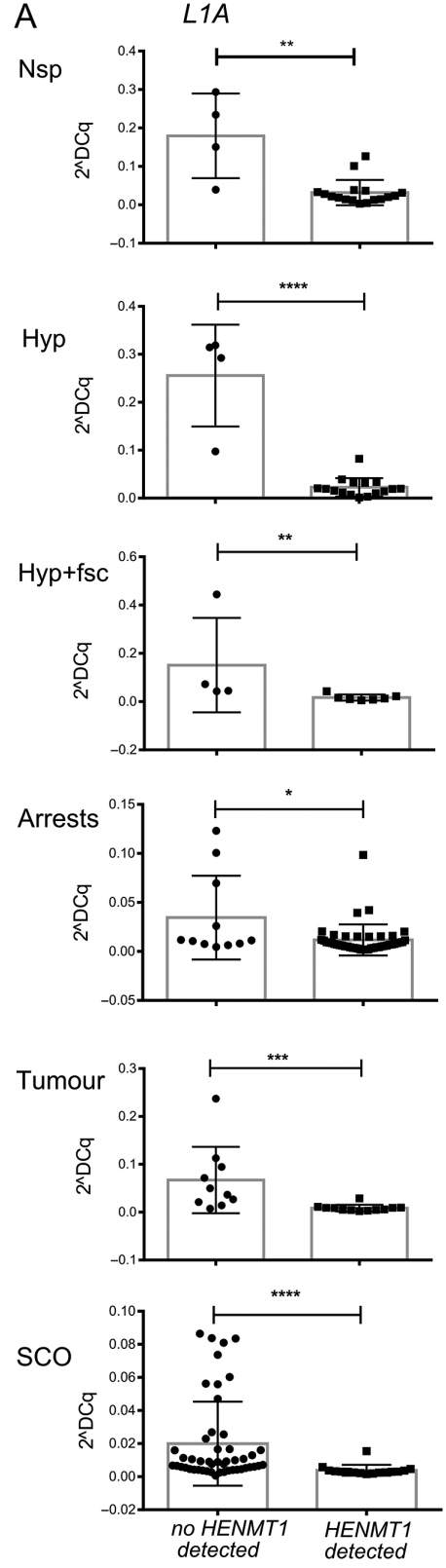

B

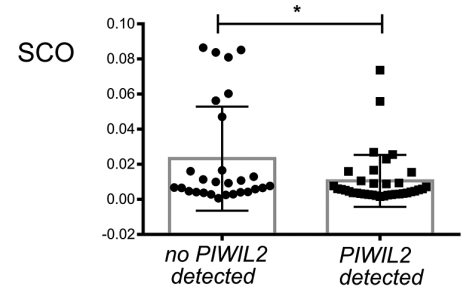

$L 1 B$
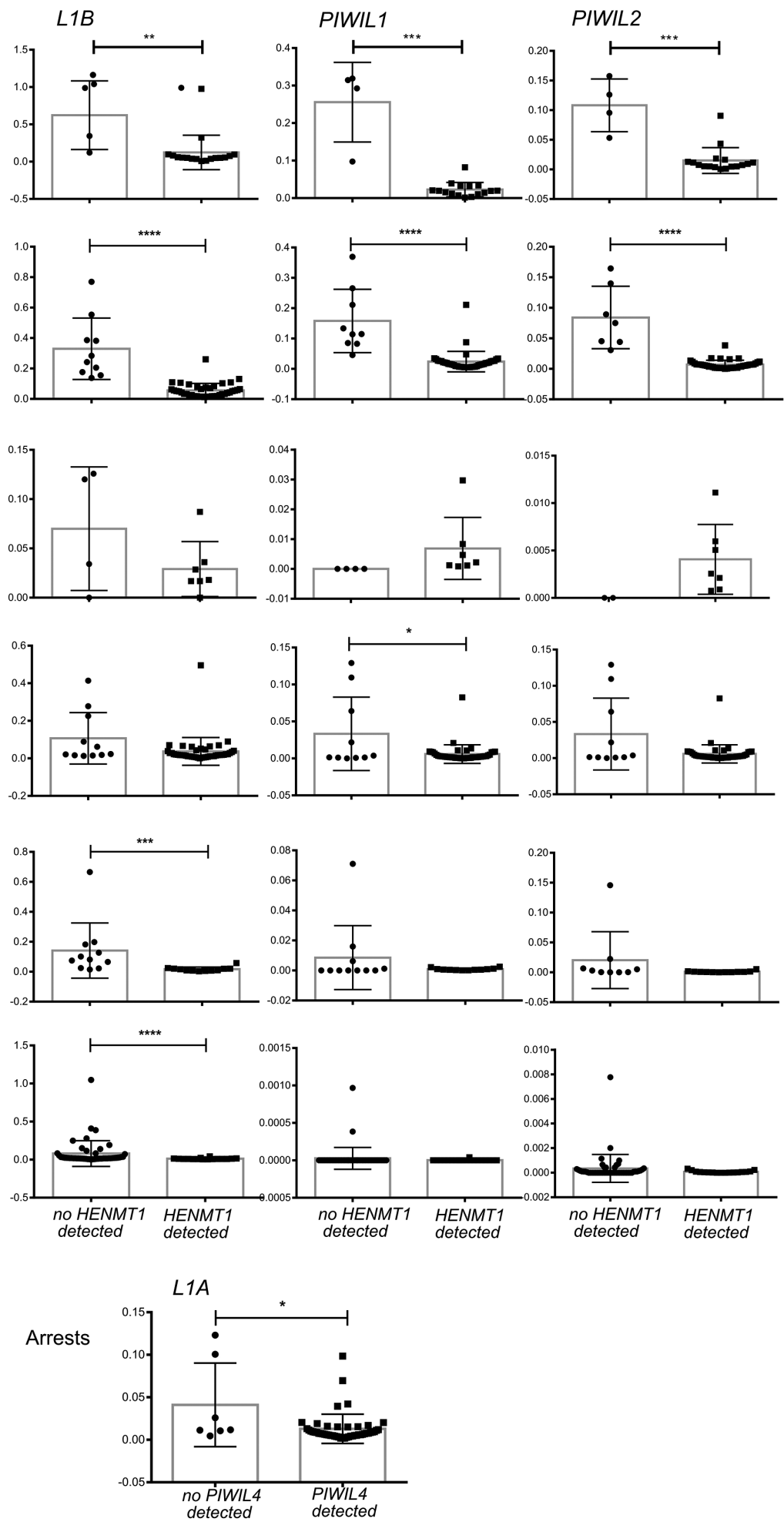

Figure 4 Loss of HENMT1 correlates with higher L1, PIWIL1 and PIWIL2 expression within the same histological group. (A) Mean ( \pm S.D.) expression (bar) of L1A, L1B, PIWIL1 and PIWIL2 (columns) in patient samples (dots) with no detected HENMT1 expression (left bar) and with detected HENMT1 expression (right bar) in the histological groups (rows). Nsp $n=4 / 17$, Hyp $n=9 / 40$, Hyp $+\mathrm{fsC}_{\mathrm{s}}=4 / 7$, Arrests $n=11 / 44$, Tumour $n=10 / 12, \mathrm{SCO}=49 / 16$. $P$ values: ${ }^{*} P \leq 0.05 ;{ }^{* *} P \leq 0.01 ;{ }^{* * *} P \leq 0.001 ; * * * P \leq 0.0001$. (B) Mean ( \pm S.D.) expression (bar) of L1A in patient samples (dots) with no detected PIWIL2 or PIWIL4 expression (left bar) and with detected PIWIL2 or PIWIL4 expression (right bar) in the histological groups $\mathrm{SCO}=49 / 16$ (left graph) and arrests (right graph) $n=28 / 37$ ). $P$ values: $* P \leq 0.05$. 

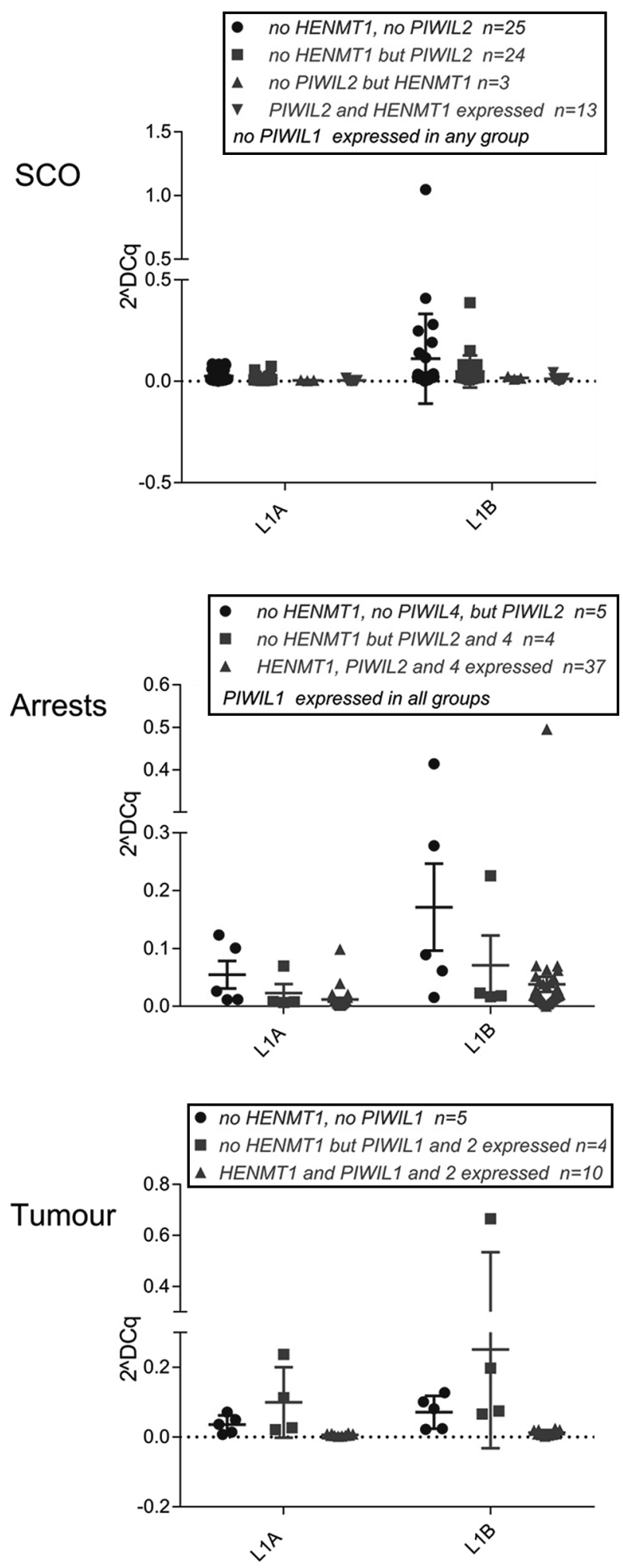

Figure 5 Transposon overexpression occurs least when both HENMT1 and PIWIL2/4 are expressed in SCO, arrest and tumour groups. Mean expression \pm S.D. (lines) and values they are derived from (dots). Legends: No HENMT1/PIWIL1/2/4=HENMT1/ PIWIL1/2/4 not detected; but HENMT1/PIWIL1/2/4 = HENMT1/ PIWIL1/2/4 detected.
L1B (ORF1) expression was lowest when both HENMT1 and PIWIL2/4 were expressed (Fig. 5) while no obvious differences in $\angle 1 A$ (5'UTR) expression were found between these subgroups.

Along the same line we wanted to know if PIWIL4 expression had an effect on the level of $L 1$ expression. This investigation was performed in the arrest group. Samples without detectable PIWIL4 expression $(n=7)$ showed a higher $L 1$ expression than samples with detectable PIWIL4 ( $n=49)$ (Fig. 4B). Samples lacking both HENMT1 and PIWIL2, or 4, showed a slightly (not significantly) higher $L 1$ expression than samples lacking HENMT1 alone (Fig. 5).

To investigate whether similar associations could be found in the tumour context, we plotted tumour samples with and without HENMT1, PIWIL1 and PIWIL2 expression against each other (Fig. 5). Samples lacking both HENMT1 and PIWIL1, but expressing PIWIL2, had only a slightly higher $L 1$ expression compared to samples expressing HENMT1, PIWIL1 and PIWIL2 which had a very low expression of $L 1$. In contrast, all samples expressing PIWIL1 and PIWIL2 without HENMT1 had an elevated expression of $L 1$.

In summary, patient samples lacking expression of a piRNA pathway component showed an elevated expression of $L 1$. Additionally, elevated $L 1$ expression coincided with significantly increased PIWIL1 and PIWIL2 expression in qualitatively complete spermatogenesis.

\section{HENMT1 overexpression leads to changes in $L 1$ expression in a seminoma cell line}

To investigate the effect of HENMT1 on transposon expression in a controlled environment, we overexpressed the full-length HENMT1 sequence in the seminoma cell line TCam2. Overexpression was performed in three independent TCam2 cell lines, and three independent TCam2 cell lines were transfected with the empty vector. HENMT1 was on average 11-fold overexpressed in the HENMT1-transfected cell lines compared to the wild-type TCam2 cells (Fig. 6A) with expression increased 15 -fold (cell line Hen 1 \#3), 13-fold (Hen1 \#4) and 8-fold (Hen1 \#5) respectively (Supplementary Fig. 2). Empty-vector-transfected cells showed no significant increase in HENMT1 expression compared to wild-type TCam2 (fold change $=1$, empty vector $\# 3 ; 1.6$, empty vector $\# 4 ; 1.2$, empty vector $\# 5$ ). There was no significant change in the expression of PIWIL1, PIWIL2 or L1B in the HENMT1-overexpressing cells (Fig. 6A). However, the expression of the $L 1 A$ sequence was significantly lower in the cell lines overexpressing HENMT1, indicating a moderating effect of HENMT1 on the expression of $L 1$ sequences, similar to what we observed in the human testis biopsies and what has been published for mouse testis (Lim et al. 2015). 


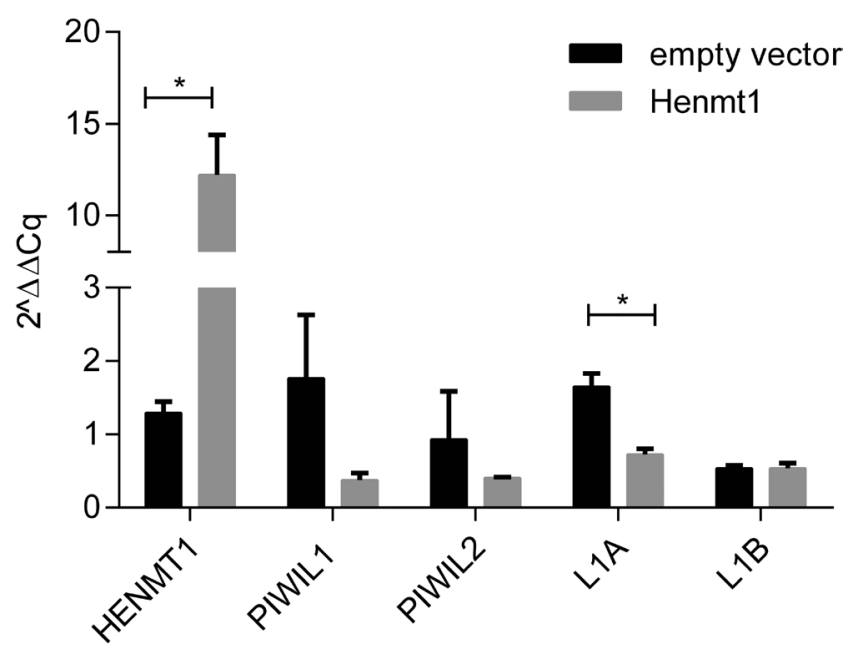

Figure 6 HENMT1 overexpression results in decreased $\angle 1 A$ expression in a seminoma cell line. Expression of piRNA pathway components (HENMT1, PIWIL1, PIWIL2) and transposon sequences $(L 1 A, L 1 B)$ in empty-vector-transfected (black) and HENMT1transfected (grey) TCam2 cell lines relative to wild-type expression. Mean \pm S.E.M. $(n=3$ cell lines for both empty-vector-transfected and HENMT1-transfected cells with three technical replicates). ${ }^{*} P \leq 0.05$.

\section{Discussion}

We have for the first time defined the exact localisation and co-localisation of HENMT1 and PIWIL1 in human testis. They were found to be co-expressed in latepachytene spermatocytes and round and elongating spermatids. HENMT1 was additionally found in spermatogonia, early spermatocytes, and at lower levels in Sertoli cells. The co-expression of HENMT1 and PIWIL1 also coincided with the expression of the pachytene piRNA population (Aravin et al. 2007, Brennecke et al. 2007, Lim et al. 2015) and matched data from the mouse (Deng \& Lin 2002, Lim et al. 2015).

A germ-cell-dominated expression pattern was supported by data gained from human testis biopsies. Namely, expression of HENMT1, PIWIL1, PIWIL2 and $L 1$ was highest in complete spermatogenesis and progressively reduced consistent with reduced germ cell content in hypospermatogenesis, germ cell arrests, tumours and SCO samples. The high $L 1$ expression in some Nsp samples was unexpected considering L1 overexpression is thought to cause abnormal spermatogenesis in mammals (Yang \& Wang 2016).

Low levels of expression could be detected for HENMT1 and PIWIL2 in the SCO group. However, Piwil2 expression in Sertoli cells has previously been reported in the mouse (Lim et al. 2013) and the PIWIL2 promoter methylation level in human SCO samples has been reported to be lower than in other somatic cell types (Heyn et al. 2012).

While the expression of HENMT1 and PIWIL2 in tumours was not unexpected, as they are normally expressed in spermatogonia or PGCs, the cells TGCT are thought to derive from, a surprising $70 \%$ of tumour samples also expressed PIWIL1 which is not normally expressed in premeiotic germ cells. This indicates an aberrant expression of PIWIL1 in many germ cell tumours. Overexpression of PIWIL1 in the TGCT context has previously been reported (Qiao et al. 2002).

The reduction of PIWIL2 expression in the arrest and tumour groups compared to Nsp was less pronounced than that for PIWIL1. This pattern is consistent with the mouse data, if we assume the PIWIL2 protein localisation is similar to the mouse where PIWIL2 is expressed from $12.5 \mathrm{dpc}$ until the round spermatid stage (Kuramochi-Miyagawa et al. 2004, Aravin et al. 2008, Manakov et al. 2015) while PIWIL1 is mainly produced in spermatocytes and spermatids which are reduced in arrests and tumour samples. Nevertheless, PIWIL2 expression was reduced in the arrest and tumour groups. PIWIL2 has been found to be hypermethylated in patients with spermatogenic failure, leading to lower PIWIL2 expression, defective piRNA biogenesis and $L 1$ hypomethylation (Heyn et al. 2012). This could be an explanation for the reduction we observed, in addition to the changes in cell population.

PIWIL3 could not be detected in human testis. It has previously been reported to be expressed in the human ovary (Lim et al. 2014) and in some cancers (Li et al. 2010, Chen et al. 2013), but has not been seen in the testis. While it has been reported that an SNP in the coding region of PIWIL3 correlates with oligozoospermia in Chinese patients (Gu et al. 2010), expression of PIWIL3 in the testis was not investigated. While PIWIL3 might be involved in testis development and not expressed in the adult testis, this could not be tested in our samples.

PIWIL4 was expressed in $91 \%$ of the samples analysed. This is not unexpected as it has been shown to be essential for prospermatogonial development in the mouse (Bao et al. 2014) and thus should be present in all samples containing spermatogonia.

$L 1$ was expressed in all samples examined, with slight differences between $\angle 1 A$ and $\angle 1 B$. Highest $L 1$ expression was found in complete spermatogenesis, and expression levels were lower in all spermatogenesisimpaired groups. In the tumour samples however, $L 1$ expression was higher in relation to the piRNA pathway components than in any other group. High $L 1$ expression in tumours could be explained considering seminoma cells are thought to be gonocyte-like cells (Sperger et al. 2011), wherein global demethylation takes place and retrotransposons may be de-repressed. In addition, cancer cells in general are prone to abnormal DNA methylation patterns and $L 1$ upregulation (Carreira et al. 2014, Koukoura et al. 2014, Rodic et al. 2014). To further complicate the story, it has been shown that some piRNA pathway components are epigenetically disrupted in a subset of human TGCTs (Ferreira et al. 2014), 
which could additionally affect retrotransposon control in the tumour context.

Unexpectedly, we discovered that $L 1 s$ could be overexpressed in individual human testes, even in those showing normal spermatogenesis. This was surprising, considering that retrotransposon control is thought to be a prerequisite for normal spermatogenesis (Kuramochi-Miyagawa et al. 2008, Reuter et al. 2011a, Zamudio et al. 2015). These data indicate that a certain level of retrotransposon expression is tolerated without obviously affecting spermatogenesis at a histological level. In support of this idea, in parallel studies where we have assessed L1 expression in mice, animals heterozygous for the Henmt1 mutation also display significantly elevated L1 expression (unpublished, MKOB). This is potentially a very significant finding as it indicates that sperm from fertile men with unrecognised L1 overexpression could transmit active L1 elements, and a potentially abnormal epigenome, to offspring with serious consequences for long-term health.

Consistent with HENMT1 having an important role in TE suppression in the human testis, mean $L 1$ levels were higher in testis biopsies without HENMT1 expression, regardless of the histological group. This association was confirmed in a controlled system provided by TCam2 seminoma cells overexpressing HENMT1, which showed significantly reduced $L 1$ expression, and is consistent with overexpression of retrotransposons in germ cells of the Henmt 1 mouse mutant due to impaired piRNA stability (Lim et al. 2015).

Our finding that $L 1 s$ were overexpressed in samples lacking expression of one or more Piwis fits well with the established models which show PIWI proteins are essential for retrotransposon control at an epigenetic and post-transcriptional levels (Kuramochi-Miyagawa et al. 2004, Reuter et al. 2011b, Manakov et al. 2015). Although this cannot be directly tested in human testis, our data supports a requirement of PIWIL1 and PIWIL2 for complete spermatogenesis in humans as their encoding mRNA was detected in all samples with complete spermatogenesis. Again, this supports a conserved function of the piRNA pathway within mammals, as both proteins have been shown to be required for mouse spermatogenesis (Deng \& Lin 2002, Kuramochi-Miyagawa et al. 2004).

Somewhat surprisingly, elevated $L 1$ expression in samples with low HENMT1 coincided with significantly increased PIWIL1 and PIWIL2 expression in qualitatively complete spermatogenesis. This increased expression of PIWIL1 and PIWIL2 is suggestive of a feedback loop wherein the instability of piRNAs, due to less methylation by HENMT1, and increased retrotransposon expression leads to the upregulation of other piRNA pathway members (Lim et al. 2015). The upregulation of PIWIL1 and PIWIL2 was only significant in the Nsp and Hyp groups, so it might be impaired in severely affected spermatogenesis, arrest and tumour samples. Alternatively, PIWIL1/PIWIL2 upregulation might occur mainly in spermatids, which are present in large numbers only in normal spermatogenesis and hypospermatogenesis.

Overall, our data supports both the presence of the $\mathrm{PIWl} /$ piRNA pathway in the human testis, and functions in the regulation of retrotransposon expression. In addition, we show that the effectiveness of this protective mechanism could be affected in a neoplastic context and in severely affected non-malignant spermatogenesis. Furthermore, we found that L1 retrotransposons were overexpressed in some human testes.

Further functional studies and a definition of the piRNA populations present in normal and abnormal spermatogenesis are needed to determine the exact mechanisms of the piRNA pathway in human testis and how they can be understood to improve clinical outcomes.

\section{Supplementary data}

This is linked to the online version of the paper at http://dx.doi. org/10.1530/REP-16-0586.

\section{Declaration of interest}

The authors declare that there is no conflict of interest that could be perceived as prejudicing the impartiality of the research reported.

\section{Funding}

Funding for this work was provided by the German Research Foundation (DFG) via the International Research Training Group (IRTG) 1871. M K O'B is funded by a National Health and Medical Research Council Australia (NHMRC) Fellowship. The work was funded in part by a grant from the Australian Research Council to M K O'B and D L A. S L L was funded by a fellowship from the Lalor Foundation (USA).

\section{Acknowledgements}

The authors gratefully acknowledge Joerg Vogelsberg, Dr Marianna Volpert, Donna Jo Merriner, Dr Daniela Fietz, Alexandra Hax and Jutta Dern-Wieloch for valuable technical assistance; Monash Microimaging facility for the provision of confocal microscopes; and Monash Biochemistry Imaging facility for provision of the 384-well cycler.

\section{References}

Aravin AA, Hannon GJ \& Brennecke J 2007 The Piwi-piRNA pathway provides an adaptive defense in the transposon arms race. Science $\mathbf{3 1 8}$ 761-764. (doi:10.1126/science.1146484)

Aravin AA, Sachidanandam R, Bourc'his D, Schaefer C, Pezic D, Toth KF, Bestor T \& Hannon GJ 2008 A piRNA pathway primed by individual transposons is linked to de novo DNA methylation in mice. Molecular Cell 31 785-799. (doi:10.1016/j.molcel.2008.09.003) 
Bao J, Zhang Y, Schuster AS, Ortogero N, Nilsson EE, Skinner MK \& Yan W 2014 Conditional inactivation of Miwi2 reveals that MIWI2 is only essential for prospermatogonial development in mice. Cell Death and Differentiation 21 783-796. (doi:10.1038/cdd.2014.5)

Bergmann M \& Kliesch S 2010 Testicular biopsy and histology. In Andrology - Male Reproductive Health and Dysfunction, pp. 155-168. Eds E Nieschlag, HM Behre \& S Nieschlag. Stuttgart: Springer.

Bourc'his D \& Bestort TH 2004 Meiotic catastrophe and retrotransposon reactivation in male germ cells lacking Dnmt3L. Nature 431 96-99. (doi:10.1038/nature02886)

Brennecke J, Aravin AA, Stark A, Dus M, Kellis M, Sachidanandam R \& Hannon GJ 2007 Discrete small RNA-generating loci as master regulators of transposon activity in drosophila. Cell 128 1089-1103. (doi:10.1016/j.cell.2007.01.043)

Carmell MA, Girard A, van de Kant HJ, Bourc'his D, Bestor TH, de Rooij DG \& Hannon GJ 2007 MIWI2 is essential for spermatogenesis and repression of transposons in the mouse male germline Developmental Cell 12 503-514. (doi:10.1016/j.devcel.2007.03.001)

Carreira PE, Richardson, SR \& Faulkner GJ 2014 L1 retrotransposons, cancer stem cells and oncogenesis. FEBS Journal 281 63-73. (doi:10.1111/febs.12601)

Chen X, Liu J, Cheng Y \& Jia D 2002 HEN1 functions pleiotropically in Arabidopsis development and acts in $\mathrm{C}$ function in the flower. Development 129 1085-1094.

Chen C, Liu J \& Xu G 2013 Overexpression of PIWI proteins in human stage III epithelial ovarian cancer with lymph node metastasis. Cancer Biomarkers 13 315-321. (doi:10.3233/CBM-130360)

Deng W \& Lin H 2002 miwi, a murine homolog of piwi, encodes a cytoplasmic protein essential for spermatogenesis. Developmental Cell 2 819-830. (doi:10.1016/S1534-5807(02)00165-X)

Di Giacomo M, Comazzetto S, Saini H, Fazio S, de Carrieri C, Morgan M, Vasiliauskaite L, Benes V, Enright AJ \& O'Carroll D 2013 Multiple epigenetic mechanisms and the piRNA pathway enforce LINE1 silencing during adult spermatogenesis. Molecular Cell 50 601-608. (doi:10.1016/j.molcel.2013.04.026)

Esnault C, Maestre J \& Heidmann T 2000 Human LINE retrotransposons generate processed pseudogenes. Nature Genetics 24 363-367. (doi:10.1038/74184)

Fawcett DW, Eddy EM \& Phillips DM 1970 Observations on the fine structure and relationships of the chromatoid body in mammalian spermatogenesis. Biology of Reproduction 2 129-153. (doi:10.1095/ biolreprod2.1.129)

Fazio S, de Bartonicek N, Di Giacomo M, Abreu-Goodger C, Sankar A, Funaya C, Antony C, Moreira PN, Enright AJ \& O'Carroll D 2011 The endonuclease activity of Mili fuels piRNA amplification that silences LINE1 elements. Nature 480 259-263. (doi:10.1038/nature10547)

Ferreira HJ, Heyn H, Garcia del Muro X, Vidal A, Larriba S, Muñoz C, Villanueva A \& Esteller M 2014 Epigenetic loss of the PIWI/piRNA machinery in human testicular tumorigenesis. Epigenetics 9 113-118. (doi:10.4161/epi.27237)

Fietz D, Geyer J, Kliesch S, Gromoll J \& Bergmann M 2011 Evaluation of CAG repeat length of androgen receptor expressing cells in human testes showing different pictures of spermatogenic impairment. Histochemistry and Cell Biology 136 689-697. (doi:10.1007/s00418-011-0871-6)

Fietz D, Ratzenböck C, Hartmann K, Raabe O, Kliesch S, Weidner W, Klug J \& Bergmann M 2014 Expression pattern of estrogen receptors $\alpha$ and $\beta$ and G-protein-coupled estrogen receptor 1 in the human testis. Histochemistry and Cell Biology 142 421-432. (doi:10.1007/s00418014-1216-z)

Girard A, Sachidanandam R, Hannon GJ \& Carmell MA 2006 A germlinespecific class of small RNAs binds mammalian Piwi proteins. Nature 442 199-202. (doi:10.1038/nature04917)

Goh WS, Falciatori I, Tam OH, Burgess R, Meikar O, Kotaja N, Hammell M \& Hannon GJ 2015 piRNA-directed cleavage of meiotic transcripts regulates spermatogenesis. Genes and Development 29 1032-1044. (doi:10.1101/gad.260455.115)

Gou L-T, Dai P, Yang J-H, Xue Y, Hu Y-P, Zhou Y, Kang J-Y, Wang X Li H \& Hua M-M et al. 2014 Pachytene piRNAs instruct massive mRNA elimination during late spermiogenesis. Cell Research 24 680-700. (doi:10.1038/cr.2014.41)

Gu A, Ji G, Shi X, Long Y, Xia Y, Song L, Wang S \& Wang X 2010 Genetic variants in Piwi-interacting RNA pathway genes confer susceptibility to spermatogenic failure in a Chinese population. Human Reproduction 25 2955-2961. (doi:10.1093/humrep/deq274)

Guo M \& Wu Y 2013 Fighting an old war with a new weapon - silencing transposons by Piwi-interacting RNA. IUBMB Life 65 739-747. (doi:10.1002/iub.1192)

Ha H, Song J, Wang S, Kapusta A, Feschotte C, Chen KC \& Xing J 2014 A comprehensive analysis of piRNAs from adult human testis and their relationship with genes and mobile elements. BMC Genomics 15545. (doi:10.1186/1471-2164-15-545)

Heyn H, Ferreira HJ, Bassas L, Bonache S, Sayols S, Sandoval J, Esteller M \& Larriba S 2012 Epigenetic disruption of the PIWI pathway in human spermatogenic disorders. PLOS ONE 7 e47892. (doi:10.1371/journal. pone.0047892)

Iliev R, Stanik M, Fedorko M, Poprach A, Vychytilova-Faltejskova P, Slaba K, Svoboda M, Fabian P, Pacik D \& Dolezel J et al. 2016 Decreased expression levels of PIWIL1, PIWIL2, and PIWIL4 are associated with worse survival in renal cell carcinoma patients. OncoTargets and Therapy 9 217-222. (doi:10.2147/OTT.S91295)

International Human Genome Sequencing Consortium 2001 Initial sequencing and analysis of the human genome. Nature 409 960-921. (doi:10.1038/35057062)

Jamsai D, Sarraj MA, Merriner DJ, Drummond AE, Jones KT, McLachlan RI \& O'Bryan MK 2011 GGN1 in the testis and ovary and its variance within the Australian fertile and infertile male population. International Journal of Andrology 34 624-632. (doi:10.1111/j.13652605.2010.01127.x)

Jorgensen N, Rajpert-De Meyts E, Graem N, Muller J, Giwercman A \& Skakkebaek NE 1995 Expression of immunohistochemical markers for testicular carcinoma in situ by normal human fetal germ cells. Laboratory Investigation 72 223-231.

Kawaoka S, Izumi N, Katsuma S \& Tomari Y 2011 3' end formation of PIWI-interacting RNAs in vitro. Molecular Cell 43 1015-1022. (doi:10.1016/j.molcel.2011.07.029)

Kirino Y \& Mourelatos Z 2007 2'-O-methyl modification in mouse piRNAs and its methylase. Nucleic Acids Symposium Series 51 417-418. (doi:10.1093/nass/nrm209)

Koukoura O, Spandidos DA, Daponte A \& Sifakis S 2014 DNA methylation profiles in ovarian cancer: implication in diagnosis and therapy (Review). Molecular Medicine Reports 10 3-9. (doi:10.3892/mmr.2014.2221)

Kuramochi-Miyagawa S, Kimura T, Ijiri TW, Isobe T, Asada N, Fujita Y, Ikawa M, Iwai N, Okabe M \& Deng W et al. 2004 Mili, a mammalian member of piwi family gene, is essential for spermatogenesis. Development 131 (4) 839-849. (doi:10.1242/dev.00973)

Kuramochi-Miyagawa S, Watanabe T, Gotoh K, Totoki Y, Toyoda A, Ikawa M, Asada N, Kojima K, Yamaguchi Y \& ljiri TW et al. 2008 DNA methylation of retrotransposon genes is regulated by Piwi family members MILI and MIWI2 in murine fetal testes. Genes and Development 22 908-917. (doi:10.1101/gad.1640708)

Lau NC 2006 Characterization of the piRNA Complex from Rat Testes. Science 313 363-367. (doi:10.1126/science.1130164)

Li L, Yu C, Gao H \& Li Y 2010 Argonaute proteins: potential biomarkers for human colon cancer. BMC Cancer 10 38. (doi:10.1186/1471 -2407-10-38)

Lim SL, Tsend-Ayush E, Kortschak RD, Jacob R, Ricciardelli C, Oehler MK \& Grutzner F 2013 Conservation and expression of piRNA pathway genes in male and female adult gonad of amniotes. Biology of Reproduction 89 1-13. (doi:10.1095/biolreprod.113.111211)

Lim SL, Ricciardelli C, Oehler MK, Tan, IMDDA, Russell D \& Grützner F 2014 Overexpression of piRNA pathway genes in epithelial ovarian cancer. PLOS ONE 9 e99687. (doi:10.1371/journal. pone.0099687)

Lim SL, Qu ZP, Kortschak RD, Lawrence DM, Geoghegan J, Hempfling A-L, Bergmann M, Goodnow CC, Ormandy CJ \& Wong L et al. 2015 HENMT1 and piRNA stability are required for adult male germ cell transposon repression and to define the spermatogenic program in the mouse. PLOS Genetics 11 e1005620. (doi:10.1371/journal.pgen.1005620)

Manakov SA, Pezic D, Marinov GK, Pastor WA, Sachidanand R \& Aravin AA 2015 MIWI2 and MILI have differential effects on piRNA biogenesis and DNA methylation. Cell Reports 12 1234-1243. (doi:10.1016/j.celrep.2015.07.036)

Nitzsche B, Gloesenkamp C, Schrader M, Hoffmann B, Zengerling F, Balabanov S, Honecker F \& Höpfner M 2012 Anti-tumour activity of 
two novel compounds in cisplatin-resistant testicular germ cell cancer. British Journal of Cancer 107 1853-1863. (doi:10.1038/bjc.2012.481)

Plotnikova A, Baranauskè S, Osipenko A, Klimašauskas S \& Vilkaitis G 2013 Mechanistic insights into small RNA recognition and modification by the HEN1 methyltransferase. Biochemical Journal 453 281-290. (doi:10.1042/BJ20121699)

Qiao D, Zeeman A-M, Deng W, Looijenga LHJ \& Lin H 2002 Molecular characterization of hiwi, a human member of the piwi gene family whose overexpression is correlated to seminomas. Oncogene 21 3988-3999. (doi:10.1038/sj.onc.1205505)

Reuter M, Berninger P, Chuma S, Shah H, Hosokawa M, Funaya C, Antony C, Sachidanandam R \& Pillai RS 2011 a Miwi catalysis is required for piRNA amplification-independent LINE1 transposon silencing. Nature 480 264-267. (doi:10.1038/nature10672)

Reuter M, Berninger P, Chuma S, Shah H, Hosokawa M, Funaya C, Antony C, Sachidanandam R \& Pillai RS 2011b Miwi catalysis is required for piRNA amplification-independent LINE1 transposon silencing. (Available at: http://www.nature.com/nature/journal/v480/ n7376/full/nature10672.html)

Rodic N, Sharma R, Zampella J, Dai L, Taylor MS, Hruban RH, lacobuzioDonahue CA, Maitra A, Torbenson MS \& Goggins M et al. 2014 Long interspersed element-1 protein expression is a hallmark of many human cancers. American Journal of Pathology 184 1280-1286. (doi:10.1016/j. ajpath.2014.01.007)

Rounge TB, Furu K, Skotheim RI, Haugen TB, Grotmol T \& Enerly E 2015 Profiling of the small RNA populations in human testicular germ cell tumors shows global loss of piRNAs. Molecular Cancer 14153. (doi:10.1186/s12943-015-0411-4)

Saito K, Sakaguchi Y, Suzuki T, Siomi H \& Siomi MC 2007 Pimet, the drosophila homolog of HEN1, mediates 2'-O-methylation of Piwi-interacting RNAs at their 3' ends. Genes and Development 21 1603-1608. (doi:10.1101/gad.1563607)

Skakkebaek NE 2016 A brief review of the link between environment and male reproductive health: lessons from studies of testicular germ cell cancer. Hormone Research in Paediatrics 86 240-246. (doi:10.1159/000443400)

Sperger JM, Chen X, Draper JS, Antosiewicz JE, Chon CH, Jones SB, Brooks JD, Andrews PW, Brown PO \& Thomson JA 2011 Gene expression patterns in human embryonic stem cells and human pluripotent germ cell tumors. PNAS 100 13350-13355 (doi:10.1073/ pnas.2235735100)

Suzuki R, Honda S \& Kirino Y 2012 PIWI expression and function in cancer. Frontiers in Genetics 3 204. (doi:10.3389/fgene.2012.00204)

Symer DE, Connelly C, Szak ST, Caputo EM, Cost GJ, Parmigiani G \& Boeke JD 2002 Human L1 retrotransposition is associated with genetic instability in vivo. Cell 110 327-338. (doi:10.1016/S00928674(02)00839-5)
Takebe M, Onohara Y \& Yokota S 2013 Expression of MAEL in nuage and non-nuage compartments of rat spermatogenic cells and colocalization with DDX4, DDX25 and MIWI. Histochemistry and Cell Biology 140 169-181. (doi:10.1007/s00418-012-1067-4)

Vourekas A, Zheng Q, Alexiou P, Maragkakis M, Kirino Y, Gregory BD \& Mourelatos Z 2012 Mili and Miwi target RNA repertoire reveals piRNA biogenesis and function of Miwi in spermiogenesis. Nature Structural and Molecular Biology 19 773-781. (doi:10.1038/nsmb.2347)

Wang H-L, Chen B-B, Cao X-G, Wang J, Hu X-F, Mu X-Q \& Chen X-B 2015 The clinical significances of the abnormal expressions of Piwil1 and Piwil2 in colonic adenoma and adenocarcinoma. OncoTargets and Therapy 8 1259-1264. (doi:10.2147/OTT.S77003)

Watanabe T, Chuma S, Yamamoto Y, Kuramochi-Miyagawa S, Totoki Y, Toyoda A, Hoki Y, Fujiyama A, Shibata T, Sado T et al. 2011 MITOPLD is a mitochondrial protein essential for nuage formation and piRNA biogenesis in the mouse germline. Developmental Cell 20 364-375. (doi:10.1016/j.devcel.2011.01.005)

Watanabe T, Cheng E-C, Zhong M \& Lin H 2015 Retrotransposons and pseudogenes regulate mRNAs and IncRNAs via the piRNA pathway in the germline. Genome Research 25 368-380. (doi:10.1101/gr.180802.114)

Webster KE, O'Bryan MK, Fletcher S, Crewther PE, Aapola U, Craig J, Harrison DK, Aung H, Phutikanit N, Lyle R et al. 2005 Meiotic and epigenetic defects in Dnmt3L-knockout mouse spermatogenesis. PNAS 102 4068-4073. (doi:10.1073/pnas.0500702102)

Williams Z, Morozov P, Mihailovic A, Lin C, Puvvula PK, Juranek S, Rosenwaks Z \& Tuschl T 2015 Discovery and characterization of piRNAs in the human fetal ovary. Cell Reports 13 854-863. (doi:10.1016/ j.celrep.2015.09.030)

Yang F \& Wang PJ 2016 Multiple LINEs of retrotransposon silencing mechanisms in the mammalian germline. Seminars in Cell and Developmental Biology 59 118-112. (doi:10.1016/j. semcdb.2016.03.001)

Yu B, Yang Z, Li J, Minakhina S, Yang M, Padgett RW, Steward R \& Chen X 2005 Methylation as a crucial step in plant microRNA biogenesis. Science 307 932-935. (doi:10.1126/science.1107403)

Zamudio N, Barau J, Teissandier A, Walter M, Borsos M, Servant N \& Bourc'his D 2015 DNA methylation restrains transposons from adopting a chromatin signature permissive for meiotic recombination. Genes and Development 29 1256-1270. (doi:10.1101/gad.257840.114)

Received 1 November 2016

First decision 6 December 2016

Revised manuscript received 8 June 2017

Accepted 4 July 2017 